\title{
Fracture Femur in a Case of Pyle's Disease: A Case Report
}

\author{
Wasudeo Gadegone, Vijayanand Lokhande \\ Department of Orthopaedics \& Traumatology, Chandrapur Multispeciality Hospital, Chandrapur, India \\ Email: gadegone123@yahoo.co.in
}

Received 23 August 2015; accepted 26 October 2015; published 29 October 2015

Copyright (C) 2015 by authors and Scientific Research Publishing Inc.

This work is licensed under the Creative Commons Attribution International License (CC BY). http://creativecommons.org/licenses/by/4.0/

c) (i) Open Access

\begin{abstract}
Pyle's disease is a rare genetic skeletal disorder also known as Metaphyseal dysplasia. A case of 36 yrs old female with fracture supracondylar femur in Pyle's disease is presented. She had mild facial dysmorphism, dental malformation \& mild genu valgum. Skeletal radiology revealed the characteristic Erlenmeyer flask sign at distal femoral and proximal tibial metaphysis. The fracture supracondylar femur was treated with interlocking nail. The fracture was united in sixteen weeks period. The patient is followed up for two years. The aim of the case report is the rarity of the condition with fracture supracondylar femur in adult female successfully treated with interlocking nail.
\end{abstract}

\section{Keywords}

Pyle's Disease, Metaphyseal Dysplasia, Erlenmeyer Flask Sign, Fracture Femur, Interlocking Nailing

\section{Introduction}

Metaphyseal dysplasia (also known as Pyle disease) is a rare autosomal recessive disorder characterised by flaring of the ends of long bones with relative constriction and sclerosis of the diaphysis and mild cranial sclerosis. It is one of the causes of an Erlenmeyer flask deformity. The flared metaphases are relatively lucent. The patients are often asymptomatic although genu valgus deformity may be a feature. The disorder was first reported by Pyle in 1931 as "a case of unusual bone development" [1]. Bakwin and Krida restudied the same case and his affected sister and designated the disorder "familial metaphyseal dysplasia” [2]. When there is cranial involvement, the term craniometaphyseal dysplasia was used. Its confusion with craniometaphyseal dysplasia was clarified by Gorlin et al. [3]. Finally, the term "metaphyseal dysplasia” was used in the 1983 in Paris, and the eponym Pyle was added in brackets. The aim of this paper is to present a case of Pyle's disease with fracture femur success- 
fully treated by interlock nail.

\section{Case Report}

The 36 yrs old female came to us with pain, swelling, deformity and shortening of right femur after trivial fall while working in the field. She is 5.2 feet tall with average built with no history of diabetes, hypertension or other comorbidities. The mental status was normal for her age (Figure 1). There was no history of bone or joint pains, anaemia, jaundice, blood transfusion or developmental delay. There was no family history of similar problem. On examination it was found that patient has deformity of right femur, pain and swelling. She had mild facial dysmorphism, mild prognathism, genu vulgum deformity, kyphotic deformity of spine and restricted extension of bilateral elbow joints with pronated both forearms, she was having dental malocclusion. The hip, shoulder and wrist examination were normal. Cranial nerve examination was normal. Examinations of respiratory, cordiovasclular systems were normal. Examination of the parents and her two sisters was normal.

In our case radiography shows fracture at supracondylar femur on right side (Figure 2). Skeletal survey showed both knee joints revealed uniform broadening of bilateral metaphysis of proximal distal femur \& proximal tibia showing Erlenmeyer flask sign (Figure 3). Multiple growth arrest lines, diffuse osteopenia was also seen .There was broadening of metaphysis of other long bones. Proximal two-thirds of humerus and distal two-thirds of radius and ulna show similar changes (Figure 4). X-ray of the dorsolumbar spine revealed platyspondyly. X-ray skull, cervical spine and chest were normal. Haemogram, biochemical, serological investigations were within normal limits except low vit D3 level. Based on clinical and radiological findings, we made a diagnosis of fracture supracondylar femur in a patient of Pyle's metaphyseal dysplasia.

Surgery was performed on third day of injury. Under spinal analgesia on operation table the fracture was reduced. Two cm patellar tendon splitting incision is given. Entry is made in the lower supracondylar notch. The guide wire is passed and over the guide wire reaming was done. The close supracondylar nailing with static locking was done. The postoperative course was uneventful. Fracture has united in 16 weeks. Patient is followed up to 2 years (Figure 5). There is restriction of terminal range of flexion of knee. At final follow-up patient is able to perform indoor and outdoor activities without pain. No treatment was given for Pyle's disease as patient did not have any complaints.

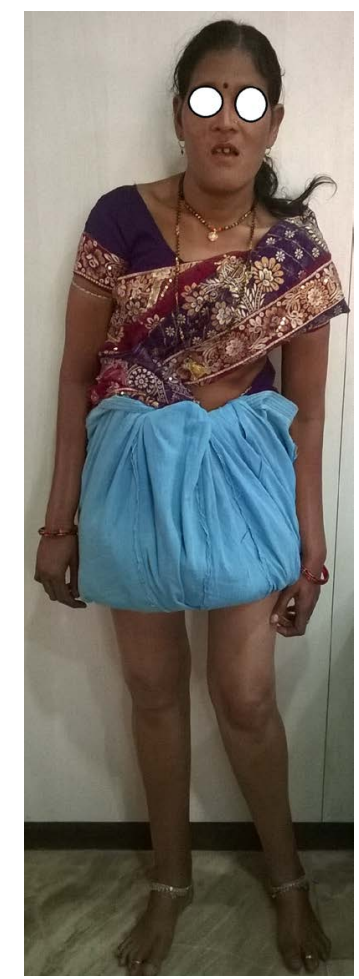

Figure 1. Clinical photograph of 36 years old female patient of Pyle’s disease. 


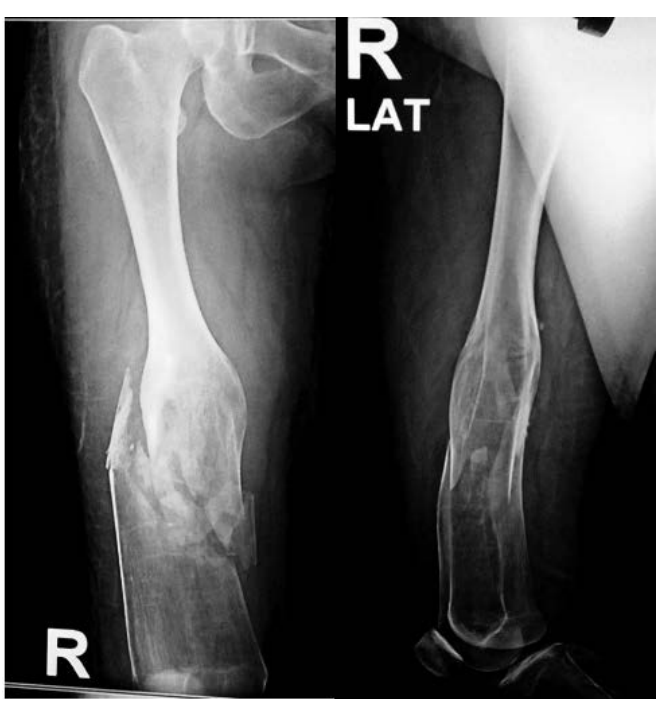

Figure 2. 36 yrs old female with supracondylar fracture femur. Preoperative X-ray Ap and lateral.
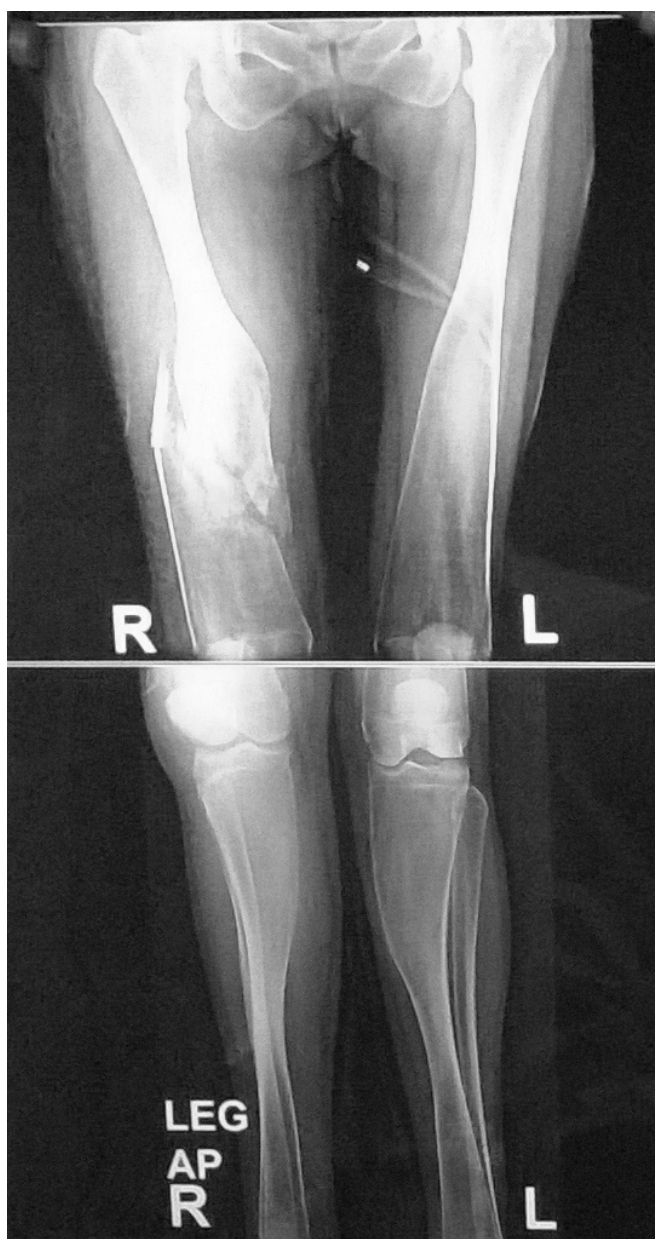

Figure 3. 36 years old female patient Radiography of both knee joints revealed uniform broadening of bilateral metaphysis of proximal distal femur \& proximal tibia showing Erlenmeyer flask sign. 


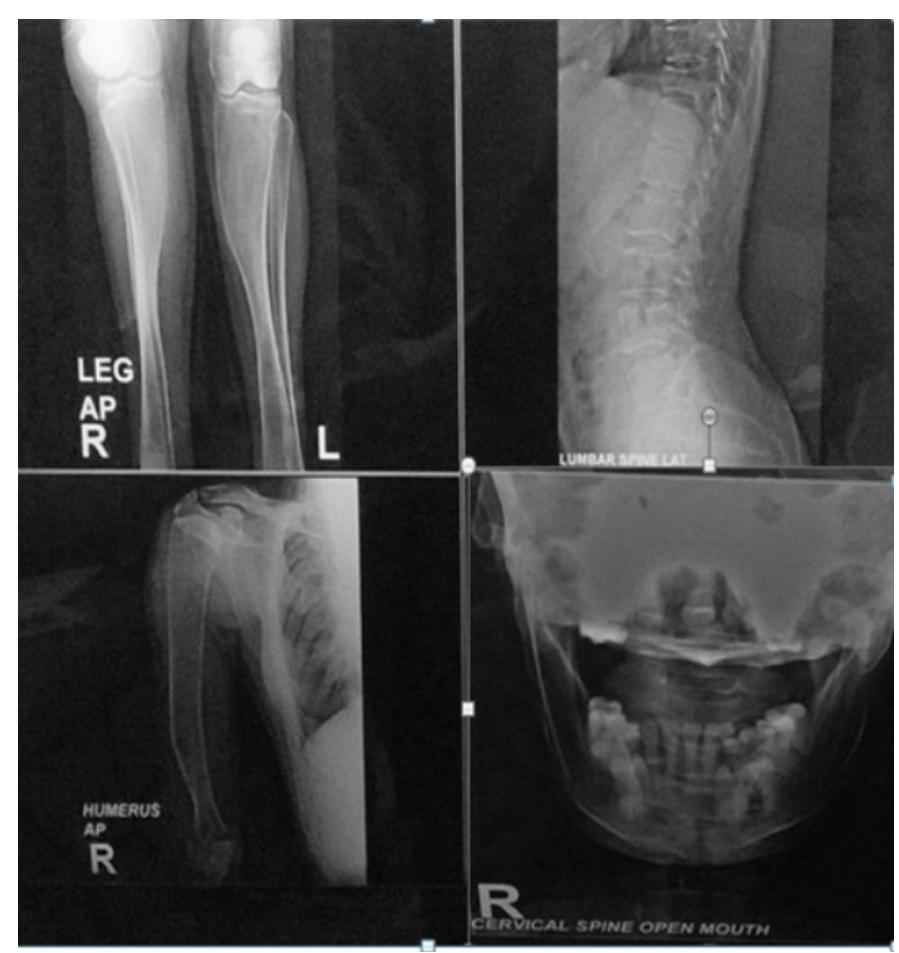

Figure 4. Radiological features in other bones showing Flaring and widening of metaphysis, mild jaw involvement and platyspondyly of lower thoracic vertebra.

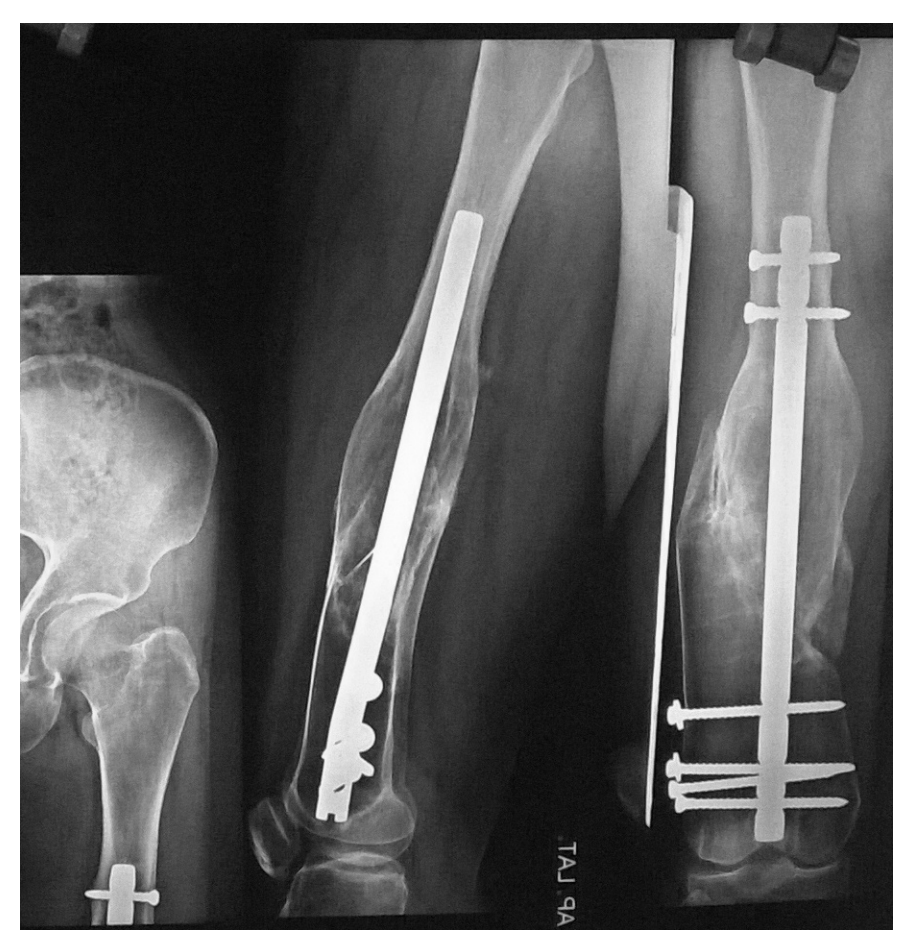

Figure 5. Good union of fracture 2 yrs follow-up.

\section{Discussion}

There are considerable numbers of disorders both genetic \& acquired, which are associated with metaphyseal 
flaring [4]. Pyle's disease is a rare disorder with striking radiographic manifestations in contrast with the relatively normal clinical features. It has an autosomal recessive inheritance. It is characterized by defect in metaphyseal remodeling that leads to grossly widened metaphyses of long bones with marked cortical thinning and osteoporosis and Erlenmeyer-flask deformity especially in the distal end of femur and proximal tibia. Proximal two-thirds of humerus and distal two-thirds of radius and ulna may show similar changes. Similar but less striking changes are seen in other distal long bones, distal metacarpals and proximal phalanges. Spinal involvement varies from moderate platyspondyly to biconcave lens appearance of the vertebral bodies [3] [4]. Jaw involvement has also been documented [5]. Along with these roentgenographic changes, there may be few clinical signs and symptoms like muscle weakness, joint pain, genu valgum, scoliosis, and limited extension of elbow. Other differential diagnoses of Erlenmeyer flask deformity are craniotubular dysplasias including craniometaphyseal and craniodiaphyseal dysplasia, Gaucher disease, osteopetrosis, thalassemia and Niemann Pick disease. It should be differentiated from craniometaphyseal dysplasia, which is characterised by occult hypertelorism and broadening of the base of nose, increased bone sclerosis and often narrowing of neural foramina leading to mixed type deafness. Absence of cranial involvement excludes first three diagnoses in our patient [6]. Moreover, our patient did not have anemia, jaundice, hepatosplenomegaly, increased bone density or any history of blood transfusion ruling out the later. Radiographically flaring of metaphysis is greater in Pyle's disease than craniometaphyseal dysplasia. In our case the skull is only mildly involved. There is supraorbital bulge and mild prognathism. Moreover, this patient performs her daily routine work without any functional disability. Surgical and medical treatment is rarely required if the patient is asymptomatic. Symptomatic progressive genu valgum requires surgical intervention. Temporary asymmetrical tibial epiphysiodesis in children or bilateral osteotomies in adults can correct the progressive genu valgum [7]. There is somewhat greater tendency to fracture the bones. This patient suffered fracture femur while working in the field. In our case supracondylar fracture was treated by interlocking nailing. During surgery there was no problem of anaesthesia. Postoperative course was uneventful. The fracture was united without any complication in stipulated time.

\section{Conclusion}

We are reporting a rare case of Pyle's disease with fracture supracondylar femur with two years follow up without any complication.

\section{References}

[1] Pyle, E. (1931) A Case of Unusual Bone Development. Journal of Bone and Joint Surgery, 13, 874-876.

[2] Bakwin, H. and Krida, A. (1937) Familial Metaphyseal Dysplasia. American Journal of Diseases of Children, Chicago, 53, 1521-1527.

[3] Gorlin, R.J., Spranger, J. and Koszalka, M.F. (1969) Genetic Craniotubular Bone Dysplasias and Hyperostoses; A Critical Analysis. Birth Defects, 5, 79-95.

[4] Gorlin, R.J., Koszalka, M.F. and Spranger, J. (1970) Pyle’s Disease (Familial Metaphyseal Dysplasia). Journal of Bone and Joint Surgery, 52, 347-353.

[5] Beighton, P. (1987) Pyle Disease (Metaphyseal Dysplasia). Journal of Medical Genetics, 24, 321-324.

[6] Heselson, N.G., Raad, M.S., Hamersma, H., Cremin, B.J. and Beighton, P. (1979) The Radiological Manifestations of Metaphyseal Dysplasia (Pyle Disease). British Journal of Radiology, 52, 431-440.

[7] Ferrari, D., Magnani, M. and Donzelli, O. (2005) Pyle’s Disease. A Description of Two Clinical Cases and a Review of the Literature. Chir Organi Mov, 90, 303-307. 